\title{
Research on Optimization Method of Tool Path in Five-Axis Process Singular Region
}

\author{
Yujie WANG ${ }^{1}$, Xin SHEN, Yu PENG and Lixin ZHAO \\ CAC Chengdu Aircraft Industrial (Group) Co. Ltd., Chengdu 610092, China
}

\begin{abstract}
For the five -axis machine into the singular region in the process of parts processing, resulting in a discontinuous and rapid rotation of the axis of rotation of large angles. Based on the analysis of the cause of the obvious ripple on the machined surface and the influence on the machining precision, a mathematical model of the singular region is established, and an optimization method of the tool path in the singular region is proposed. The simulation and practical machining results show that the method can effectively overcome the problem of excessive movement of the rotating shaft in the Song singular region of 5-axis machine tool, and solve the surface corrugated defects caused by the problem, while improving the processing efficiency.
\end{abstract}

Keywords. Digital control process, Singular region, Tool path optimization

\section{Introduction}

The shape of aircraft structural parts is related to the aerodynamic layout of the aircraft, and the machining quality of the shape is closely related to the aerodynamic performance. Therefore, most of the contour surfaces of aircraft structural parts are curved, which need five -axis NC machine tools to complete.

The five-axis machining adds two rotating shafts to the three-axis machining, which makes the machining mode more flexible, the material removal rate is higher, the processing time is shorter, and the more complex parts can be dealt with. Therefore, five-axis machining has been widely used in aviation, aerospace, automobile, ship and other industrial fields. But the motion of rotation axis also makes the attitude control of tool more complex, which introduces many special problems of five axis machining. The singular point problem is an important one. When the tool passes through the region near the singular point, the rotation axis will produce discontinuous and rapid rotation, which greatly increases the non-linear error, and it is easy to destroy the workpiece, and even damage the machine parts.

Because of the existence of singular region, when the five -axis $\mathrm{NC}$ machining is carried out, it is easy to appear in a very short cutting length, the rotating axis rotates rapidly and discontinuously, and the variation is very large, which will produce obvious ripples on the machined surface, resulting in a great increase in the amount of grinding and grinding difficulty of the subsequent fitters, which seriously affects the

${ }^{1}$ Corresponding Author: Yujie Wang, CAC Chengdu Aircraft Industrial (Group) Co., Ltd., Chengdu China; E-mail: wyj410245590@163.com. 
machining quality of the parts and reduces the machining efficiency of the parts [1], as shown in Figure 1.

Therefore, it is very important to optimize the tool path in the singular region for improving the machining accuracy and efficiency [2]. In this paper, the cutter axis vector in the singular region is taken as the research object to solve the problem that the motion of rotation axis in the singular region is too large. In order to improve the machining accuracy and efficiency of five axis machine tool, the relevant research is carried out by means of theoretical analysis, mathematical calculation, computer programming and experimental verification. Finally, the tool path optimization software is formed, and the optimization method is verified by simulation analysis and trial cutting. The processing quality and efficiency are improved synchronously.

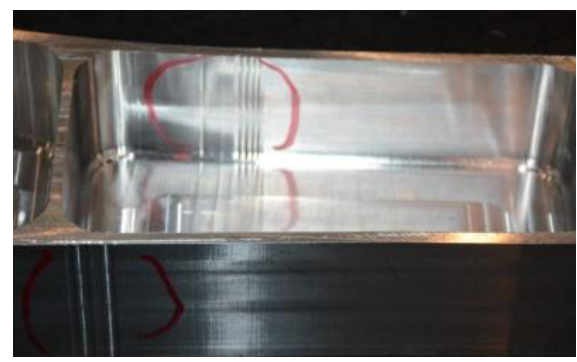

Figure 1. Surface ripple caused by excessive movement of the axis of rotation.

\section{Theoretical study on the optimization method of tool path in singular region}

Singular region is characterized by very large changes in the axis of rotation when the tool-axis vector changes very little one-dimensional angle [3]. Therefore, through the establishment of singular region tool path optimization mathematical model, analysis of the surface profile error as a guide to calculate the tool axis variation tolerance, The optimization vector is found in the tolerance region to minimize the angle between the starting vector and the target vector on the projection plane, so that the motion of the rotation axis in the singular region is minimized.

\subsection{Singularities in 5 Axis Machine Tools}

In 5-axis NC machining the so-called singular problem arises when there is inverse kinematics singular point in the machining space [4]. The inverse kinematics problem of machine tool can be expressed as solving the coordinates of each moving axis of the machine tool under the condition of known coordinate value of tool tip in workpiece coordinate system and vector direction of cutter axis. Take the AC double turntable five-axis machine tool as an example. The solution of $\mathrm{A}$ and $\mathrm{C}$ angles is obtained from the inverse kinematical transformation as follows [5]:

$$
\left\{\begin{array}{c}
A=k_{A} \bullet \alpha \cos (k), k_{A}= \pm 1 \\
C=-\arctan \left(\frac{i}{j}\right)+k_{C} \bullet \pi, k_{C}=0,1,2
\end{array}\right.
$$


where $\mathrm{i}, \mathrm{j}, \mathrm{k}$ is tool vector, $\mathrm{A}$ is in $\left[-90^{\circ}, 90^{\circ}\right], \mathrm{C}$ is in $\left[0^{\circ}, 360^{\circ}\right]$. The value of $\mathrm{A}, \mathrm{C}$ in Table 1 and Table 2 based on Eq. (1).

Table 1. AC corner numerical value $\left(\mathrm{A} \in\left[-90^{\circ}, 0^{\circ}\right]\right)$.

\begin{tabular}{ccccccc}
\hline & $\mathrm{i}$ & $>0$ & $<0$ & $<0$ & $>0$ & $=0$ \\
\cline { 2 - 6 } $\mathrm{A} \in\left[-90^{\circ}\right.$, & $\mathrm{j}$ & $>0$ & $>0$ & $<0$ & $<0$ & $>0$ \\
$\left.0^{\circ}\right]$ & $\mathrm{C}$ & $2 \pi-\arctan (\mathrm{i} / \mathrm{j})$ & $-\arctan (\mathrm{i} / \mathrm{j})$ & $\pi-\arctan (\mathrm{i} / \mathrm{j})$ & $\pi-\arctan (\mathrm{i} / \mathrm{j})$ & $2 \pi$ \\
\cline { 2 - 7 } & $\mathrm{i}$ & $=0$ & $>0$ & $<0$ & $=0$ & \\
\cline { 2 - 7 } & $<0$ & $=0$ & $=0$ & $=0$ \\
\hline $\mathrm{j}$ & $\pi$ & $3 \pi / 2$ & $\pi / 2$ & Can't be sure. \\
\hline
\end{tabular}

Table 2. AC corner numerical value $\left(\mathrm{A} \in\left(0^{\circ}, 90^{\circ}\right]\right)$.

\begin{tabular}{ccccccc}
\hline & $\mathrm{i}$ & $>0$ & $<0$ & $<0$ & $>0$ & $=0$ \\
\cline { 2 - 6 } & $\mathrm{j}$ & $>\mathbf{0}$ & $>0$ & $<0$ & $<0$ & $>0$ \\
\cline { 2 - 7 }$\left(0^{\circ}\right]$ & $\mathrm{C}$ & $\pi / 2-\arctan (\mathrm{i} / \mathrm{j})$ & $\pi-\arctan (\mathrm{i} / \mathrm{j})$ & $2 \pi-\arctan (\mathrm{i} / \mathrm{j})$ & $-\arctan (\mathrm{i} / \mathrm{j})$ & $\pi$ \\
\cline { 2 - 7 } & $\mathrm{i}$ & $=0$ & $>0$ & $<0$ & $=0$ & $=0$ \\
\cline { 2 - 7 } & $\mathrm{j}$ & $<0$ & $=0$ & $3 \pi / 2$ & Can't be sure. \\
\hline
\end{tabular}

From the Table 1, it can be seen that there is no singular problem in the solution of angle $A$, but when the solution of angle $c$ is in $i=0$ and $j=0$, the solution of $c=\pi-\arctan$ $(0 / 0)$ or $\mathrm{C}=-\arctan (0 / 0)$ has no solution, so it is impossible to determine the value of $\mathrm{c}$, that is to say, it is singular point. At this time, the turntable of the machine tool and the tool shaft are vertical, no matter what the value of the $\mathrm{C}$ angle does not affect the direction of the tool axis of the point, that is, the $\mathrm{c}$ axis can swing at any angle to obtain the tool axis vector at the singular point position, so for the AC double turntable fiveaxis machine, the point of the tool axis vector is the singularity point, and the normal axis of the $\mathrm{C}$ turntable is the singular axis.

In fact, When the angle between the tool axis vector and the singular axis is less than a certain angle, the angle of the rotation axis will change more and more, resulting in large error [6]. This angle is singular value, and the area formed by it is a cone area in space, as shown in the Figure 2.

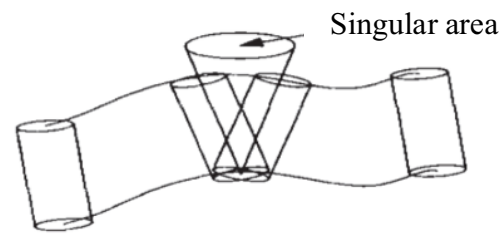

Figure 2. Schematic diagram of singular regions.

\subsection{Mathematical Modeling of Singular Regions}

According to the definition of singular region, the geometric model of the singular region is established, as shown in Figure 3.

In Figure 3, $V_{1}$ and $V_{2}$ represent the starting vector and target vector of tool axis motion, respectively, the angle between the starting vector and the target vector on the 
projection plane, $\mathrm{O}$ for the origin, $\mathrm{V}_{\mathrm{p}}$ for the polar axis, $\mathrm{V}_{\mathrm{p}}$ as the axis, $\mathrm{V}_{1}$ and $\mathrm{V}_{2}$ vectors as the busbar as the conical surface, and projection to the plane as circle $\mathrm{A}$.

When the angle between $V_{1}, V_{2}$ and polar axis $V_{p}$ is very small, even if the angle between $V_{1}$ and $V_{2}$ is very small, But the angle $\Delta \beta$ of their projection on the plane of the polar axis $V_{p}$ as the normal vector may be very large. This is the reason why the tool axis vector on the same surface changes very little, but the machine tool rotation axis movement still needs to swing substantially.

Secondly, after understanding the problems existing in the singular region, in order to reduce the movement of the rotation axis, the tool path in the singular region must be optimized. The optimization principle is to establish the mathematical model of the tool path optimization, as shown in Figure 4. The results show that the variation tolerance of the tool axis is calculated by using the machining surface profile error as the guide, and the optimal vector is found in the allowable region to minimize the $\Delta \beta$, so as to minimize the movement of the rotation axis in the singular region.

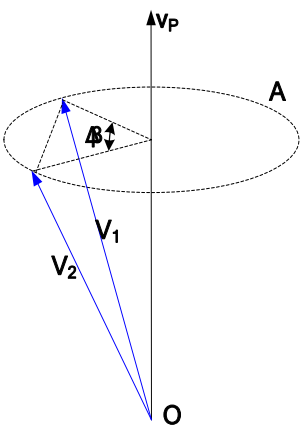

Figure 3. The geometric model of singular region.

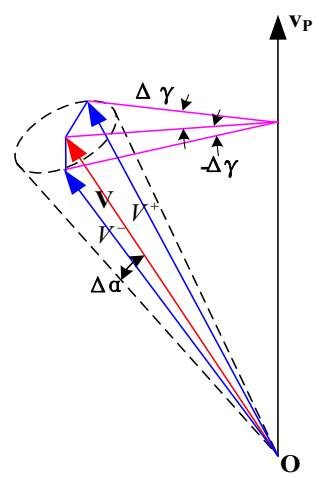

Figure 4. Geometric model of tool path optimization.

In Figure 4, the vector $\mathrm{V}$ to be optimized is used as the center line of rotation, the origin $\mathrm{O}$ is taken as the item point, and the tolerance $\Delta \alpha$ as the half-top angle as the conical face. The angles between the bus and the vector $\mathrm{V}$ of the conical surface is $\Delta \alpha$.The super polar axis $\mathrm{V}_{\mathrm{p}}$ does two planes tangent to the conical surface, and the tangent vectors are represented by vectors $V^{-}$and $V^{+}$.

The projection angles of $V^{-}$and $V^{+}$on the plane where the polar $\mathrm{V}_{\mathrm{p}}$ is the normal vector are $\Delta \gamma$ and $-\Delta \gamma$, respectively. $\mathrm{V}$ and $V^{-}, V^{+}$make plane $\mathrm{p}^{\prime}$ and $\mathrm{p}^{\prime \prime}$ respectively. The previous tool axis vector is used as the optimization objective vector of the current tool axis, and the included angle $\theta$ of the projection between the optimization objective vector and the vector $\mathrm{V}_{\text {TARGET }}$ to be optimized on the plane where the polar axis $V_{p}$ is the normal vector is calculated.

Assuming that $\mathrm{V}$ is the vector optimized by the cutter axis, it can be divided into four cases by geometric judgment

Case 1: $\theta<-\Delta \gamma$ is $V_{y}=V^{-}$;

Case 2: $\theta>\Delta \gamma$ is $V_{y}=V^{+}$; 
Case 3: $0<\theta<\Delta \gamma, V_{y}$ is $\mathrm{p}^{\prime \prime}$ with the intersection of the target vector $\mathrm{V}_{\text {TARGET }}$ and the plane of the polar axis.

Case 4: $-\Delta \gamma<\theta<0, V_{y}$ is $\mathrm{p}^{\prime}$ with the intersection of the target vector $\mathrm{V}_{\text {TARGET }}$ and the plane of the polar axis.

\subsection{Tool Path Optimization Method}

Generally, the motion path of five axis CNC machine tool (tool path) is obtained by CAM software, and the running NC program can be recognized by post-processing software. We can optimize the tool path through post-processing software to solve the problem of singular region. According to the mathematical model of tool path optimization in singular region, the flow diagram of tool path optimization is shown in Figure 5.

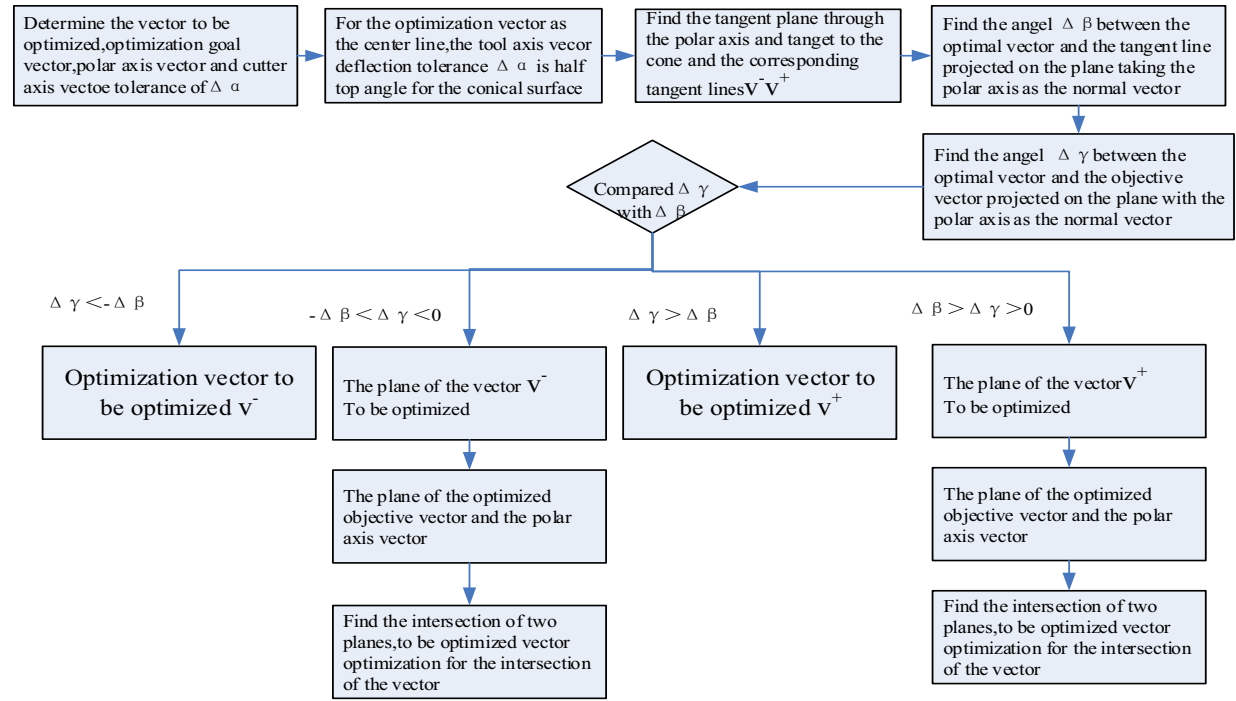

Figure 5. the flow diagram of tool path optimization.

Know: Optimize tool vector $v_{1}=\left(\begin{array}{lll}i_{1} & j_{1} & k_{1}\end{array}\right)$, optimizing vector $v=\left(\begin{array}{lll}i & j & k\end{array}\right)$, axis vector $v_{p}=\left(\begin{array}{lll}i_{p} & j_{p} & k_{p}\end{array}\right)$, tolerance $\Delta \alpha$

Find: Optimize the cutter axis vector $v_{y}=\left(\begin{array}{lll}i^{\prime} & j^{\prime} & k^{\prime}\end{array}\right)$, make the angle between the tool axis vector and the vector to be optimized $\left(v_{p}, v\right)<\Delta \alpha$, and projected on the plane with the polar vector as the normal vector of the objective optimization vector $\left|\operatorname{Ang}\left(v_{y} \quad v_{1} \quad v_{p}\right)\right|$.

By the above analysis, the model can be reduced to a three-dimensional quadratic system of equations. 


$$
\left\{\begin{array}{c}
\left(\begin{array}{l}
i \\
j \\
k
\end{array}\right) \bullet\left(\begin{array}{l}
i^{\prime} \\
j^{\prime} \\
k^{\prime}
\end{array}\right)=\cos \alpha \\
\left.\left.\left(\begin{array}{l}
i \\
j \\
k
\end{array}\right) \times\left(\begin{array}{l}
i^{\prime} \\
j^{\prime} \\
k^{\prime}
\end{array}\right)\right) \cdot\left(\begin{array}{l}
i_{p} \\
j_{p} \\
k_{p}
\end{array}\right) \times\left(\begin{array}{l}
i^{\prime} \\
j^{\prime} \\
k^{\prime}
\end{array}\right)\right)=0 \\
\left|\left(\begin{array}{l}
i^{\prime} \\
j^{\prime} \\
k^{\prime}
\end{array}\right)\right|=1
\end{array}\right.
$$

Put Eq. (2) simplify

$$
\left\{\begin{array}{c}
i i^{\prime}+j j^{\prime}+k k^{\prime}=\cos \alpha \\
\left(j k^{\prime}-k j^{\prime}\right) i_{p}+\left(k i^{\prime}-i k^{\prime}\right) j_{p}+\left(i j^{\prime}-j i^{\prime}\right) k_{p}=0 \\
i^{\prime 2}+j^{\prime 2}+k^{\prime 2}=1
\end{array}\right.
$$

From the Eq. (3) the optimized polar axis of the cutter axis vector can be obtained by the mathematical calculation $v_{y}=\left(\begin{array}{lll}i^{\prime} & j^{\prime} & k^{\prime}\end{array}\right)$.

\section{Software Development and Software Testing of Tool Path Optimization in Singular Region}

In order to realize the automatic optimization of tool path, reduce the difficulty of calculation, improve the working efficiency, and facilitate the engineering application, this paper develops the tool path optimization processing software based on VB.NET language, and verifies that the function of the software meets the requirements of tool path optimization by optimizing the preprogram of typical parts.

\subsection{Software Development}

The software input is the tool path file processed by CAM. The tool axis vector in the file is identified line by line, and the tool axis vector (target vector) meeting the optimization conditions is identified as 0 . Then read the tool axis vector marked as 0 for optimization. The tool axis vector after optimization is judged. If the tool axis vector meets the requirements of optimization objectives, the tool axis vector is identified as 1 . If it does not meet the requirements of optimization objectives, the optimization cycle will be carried out until the requirements of optimization objectives are met. The software running framework is shown in the Figure 6.

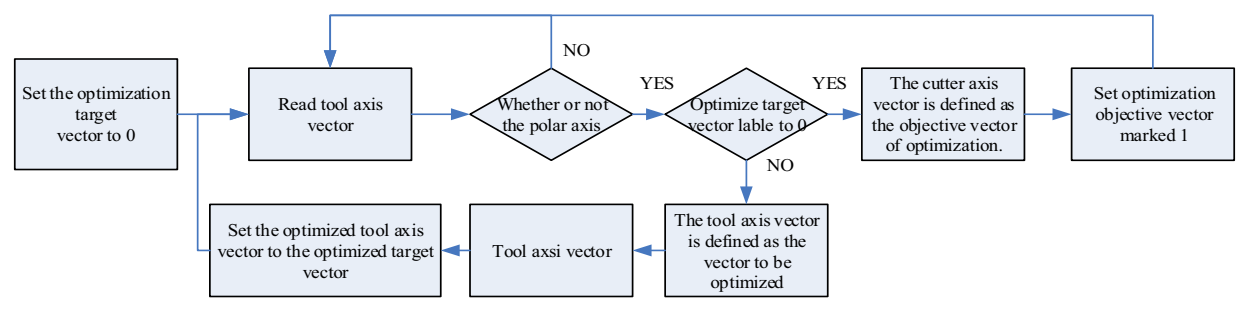

Figure 6. Block diagram of software. 


\subsection{Software Testing}

After the completion of the software, the ability test is carried out to test whether the software can effectively optimize the cutter axis vector, reduce the amount of rotation axis movement, and realize the tool of tool-path optimization method.

The "S" specimen is taken as the specimen for testing the machining accuracy of the machine tool in five axes. Its structure is shown in Figure 7. In the label area, there is a typical singular region problem, so we choose "S" pre-program to optimize the test.

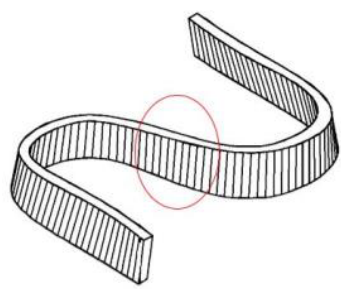

Figure 7. The schematic diagram of "S" specimen.

NC program of singular region before and after optimization are shown in Figure 8. Before the optimization, $\mathrm{C}$ angle enters and leaves from the singular region of $\mathrm{s}$ specimen, and then decreases from $113^{\circ}$ to $-34^{\circ}$. The $\mathrm{C}$ angle is transported in a short time. After optimization, the $\mathrm{C}$ angle remained almost unchanged at $-230^{\circ}$, effectively reducing the $\mathrm{C}$ angle movement.

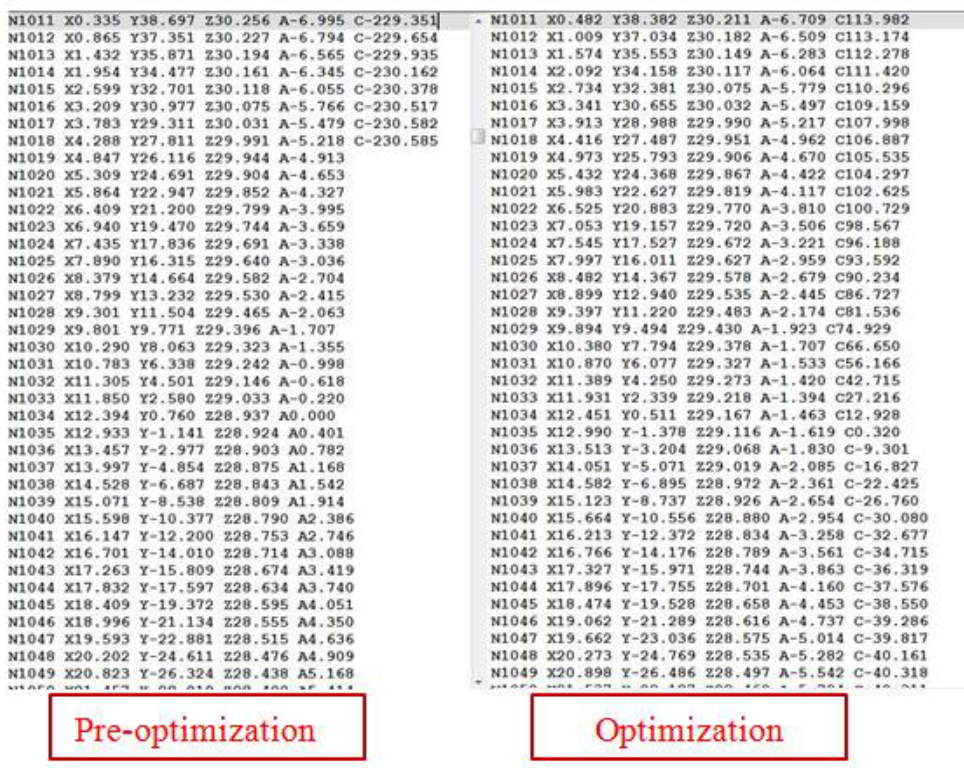

Figure 8. Comparison of Pre-optimization program and Optimization program.

Statistical optimization program C Angle travel range and C Angle total movement, the data as shown in Table 3, we can see, the range of $\mathrm{C}$ angle travel is $-234^{\circ}$ to $277^{\circ}$ before optimization and $-230^{\circ}$ to $-82^{\circ}$ after optimization. 
Table 3. "S" specimen simulation of the cutting results.

\begin{tabular}{ccc}
\hline Contrast items. & Before optimization & After optimization \\
\hline The range of C & $-234^{\circ} \sim 277^{\circ}$ & $-230^{\circ} \sim-82^{\circ}$ \\
\hline C angle exercise & $10892.916^{\circ}$ & $4669.791^{\circ}$
\end{tabular}

Therefore, after testing. the software can achieve tool path optimization algorithm, to reduce the rotation axis of the target to be.

\section{Application and Verification of Tool-Path Optimization Method in Singular Region}

The tool path optimization software is used to optimize the typical parts. Through simulation analysis and trial cutting, the machining states of the parts before and after optimization are compared to verify the optimization effect of the tool path optimization method in the singular region.

\subsection{Tool Path Optimization Simulation Analysis}

Select a typical part of the tool path optimization test verification, the simulation diagram of trial cutting part model formed by Vericut (A simulation software for NC machining) as shown in Figure 9.

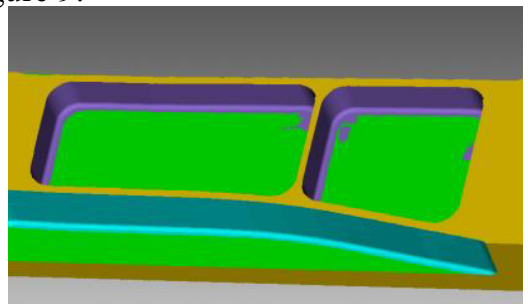

Figure 9. Simulation diagram of trial cutting part model.

The part outline program is optimized before and after the comparison, as shown in Figure 10. The tool path file processed by CAM was imported into the software for optimization. The N2172-N2185, a typical C angle with a large variation, is selected for case study.

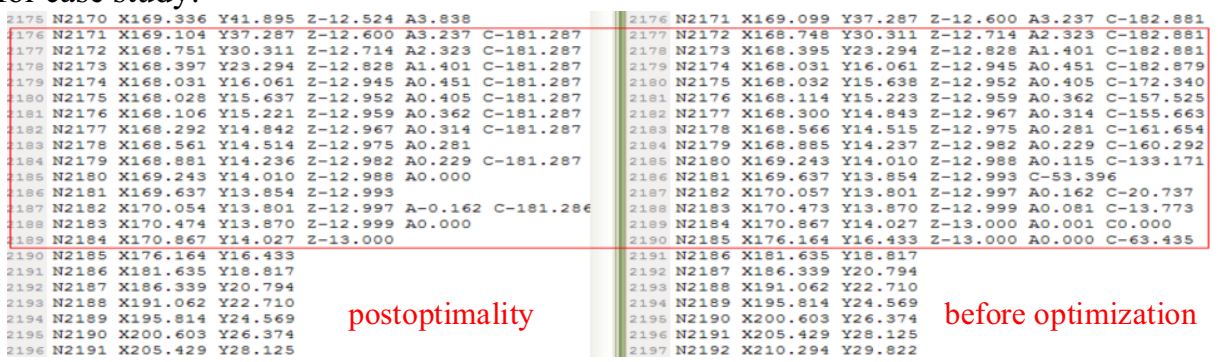

Figure 10. Comparison of actual optimization.

Before optimization, the $\mathrm{C}$ angle of the program changes rapidly from $-182.8^{\circ}$ to $\mathrm{C}^{\circ}$. After optimization, the range of $\mathrm{C}$ angle variation is very small, and there is no 
change at all. Therefore, the optimization of $\mathrm{C}$ angle variation in the optimization process is basically the same as that in $\mathrm{C}-63.4$. The effect is obvious as shown in Figure 11.

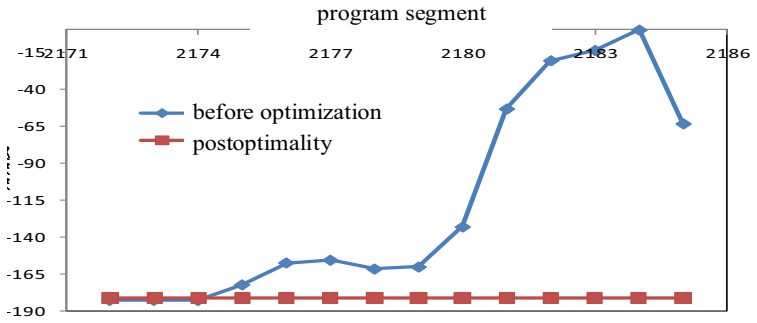

Figure 11. C angle change before and after optimization.

\subsection{Verification and Application of Cutter Path Optimization Test}

In order to verify the effectiveness of the tool path optimization method, some typical parts are tested and verified on the spot. The real-time machining time of the pre optimization and post optimization of the inner contour of the test cutting part is accurately collected by the DNC real-time monitoring module of the machine tool. The specific values are shown in Table 4.

Table 4. Comparison of actual cutting time before and after optimization.

\begin{tabular}{ccccc}
\hline & $\begin{array}{c}\text { pre-process } \\
\text { work time }\end{array}$ & $\begin{array}{c}\text { after } \\
\text { optimization } \\
\text { work time }\end{array}$ & $\begin{array}{c}\text { Shorten } \\
\text { processing } \\
\text { time }\end{array}$ & $\begin{array}{c}\text { Improve } \\
\text { processing } \\
\text { efficiency }\end{array}$ \\
\hline Contour finishing process & $270 \mathrm{~s}$ & $187 \mathrm{~s}$ & $83 \mathrm{~s}$ & $44.38 \%$ \\
\hline Inner form finishing process & $221 \mathrm{~s}$ & $123 \mathrm{~s}$ & $98 \mathrm{~s}$ & $79.67 \%$ \\
\hline
\end{tabular}

As can be seen from Table 4, the processing time before shaper optimization is $270 \mathrm{~s}$. After optimization, the efficiency is $44.38 \%$, the pre-process time is $221 \mathrm{~s}$, after optimization $123 \mathrm{~s}$, improve processing efficiency is $79.67 \%$.

The actual cutting effect is shown in Figure 12, 13 and 14. Before the optimization of the surface of the surface are corrugated, seriously affecting the surface quality, the need for benchwork follow-up grinding, but also for the final delivery of parts of the quality of filling the hidden dangers.

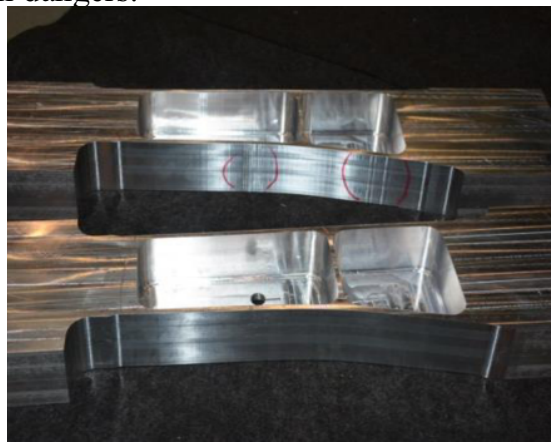

Figure 12. Comparison before and after optimization. 
After the optimization, the surface waviness has disappeared completely. The effect is good. Not only the machining efficiency is improved, but also the surface quality is greatly improved. It is verified that the optimization method of tool path in singular region can effectively solve the problem of workpiece surface waviness caused by oversized rotation axis of machine tool, and improve machining efficiency at the same time.

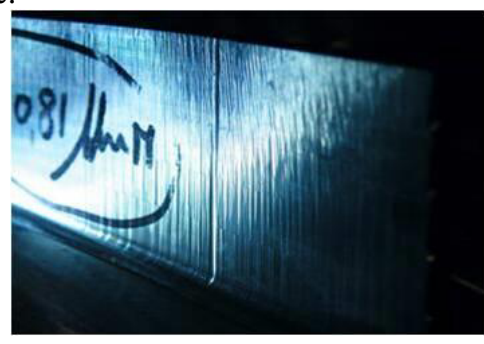

Figure 13. Surface Before Optimization

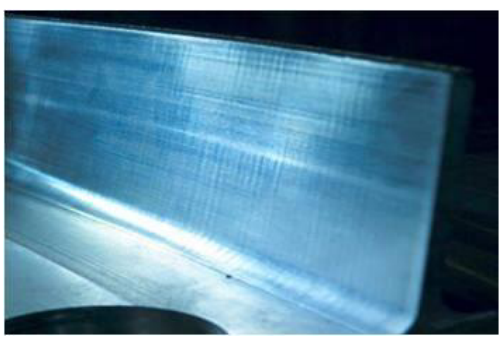

Figure 14. Surface After Optimization

\section{Conclusions and Prospects}

Based on this method, the tool path optimization software of singular region is developed. Through the simulation comparison and trial test, it is verified that this method can effectively solve the problem of workpiece surface waviness caused by the excessive rotation axis of the machine tool, and can also improve the machining efficiency significantly.

Looking ahead, as the new research project cycle is getting shorter and shorter, aircraft junction component quality requirements are getting higher and higher, five -axis NC machining applications will also be more and more, The engineering application and popularization of tool path optimization software can effectively improve the quality and efficiency of zero-part machining, so as to alleviate the pressure of production delivery and quality. We will continue to study the tool path optimization of five -axis machine tool in the future to make the tool path optimization method more reasonable and more effective, while optimizing and promoting tools software, strengthen engineering application, solve practical problems in production.

\section{References}

[1] Wang F, Lin H, Zheng LM et al. A control algorithm for rapid movements near the radius compensation singularity in five-axis end milling. J. Syst. Sci. Complexity. 2013, 26(5):718-734.

[2] Lin $\mathrm{ZW}, \mathrm{Fu} \mathrm{JZ}$, Shen HY et al. Non-singular tool path planning by translating tool orientations in C-space. Int. J. Adv. Manufacturing Tech. 2014, 71(9-12):1835-1848.

[3] Knut S. Inverse kinematics of five-axis machines near singular configurations. Int. J. Machine Tools Manufacture. 2006, 47(2):299-306.

[4] Affouard A, Duc E, Lartigue C et al. Avoiding 5-axis singularities using tool path deformation. Int. J. Machine Tools Manufacture. 2003, 44(4):415-425.

[5] Yang JX, Yusuf A. Generalized kinematics of five-axis serial machines with non-singular tool path generation. Int. J. Machine Tools Manufacture. 2013,75:119-132

[6] Lin ZW, Fu JZ, Yao XH, Sun YF. Improving machined surface textures in avoiding five-axis singularities considering tool orientation angle changes. Int. J. Machine Tools Manuf., 2015, 41-49. 